A N N A L E S Annales de Bretagne et des Pays de l'Ouest

\title{
Martin Heinzelmann (dir.), L'hagiographie du haut Moyen Âge en Gaule du Nord.
}

\section{Bernard Merdrignac}

\section{(2) OpenEdition}

1 Journals

Édition électronique

URL : http://journals.openedition.org/abpo/1750

DOI : $10.4000 /$ abpo. 1750

ISBN : 978-2-7535-1482-9

ISSN : 2108-6443

Éditeur

Presses universitaires de Rennes

Édition imprimée

Date de publication : 20 juin 2001

Pagination : 161-165

ISBN : 978-2-86847-635-7

ISSN : 0399-0826

\section{Référence électronique}

Bernard Merdrignac, "Martin Heinzelmann (dir.), L'hagiographie du haut Moyen Âge en Gaule du Nord. », Annales de Bretagne et des Pays de l'Ouest [En ligne], 108-2 | 2001, mis en ligne le 20 juin 2003, consulté le 23 septembre 2020. URL : http://journals.openedition.org/abpo/1750 ; DOI : https://doi.org/ $10.4000 / a b p o .1750$ 
exemple). Bref, une agréable invitation au voyage, une incitation intelligente à la découverte, somme toute un travail utile, sans prétention excessive.

Bernard Merdrignac

Martin HeInzElmann (dir.), L'hagiographie du haut Moyen Âge en Gaule du Nord. Manuscrits, textes et centres de production, Beihefte der Francia. Herausgegeben vom Deutschen Historischen Institut Paris. Band 52, Jan Thorbecke Verlag, Stuttgart, 2001.

Nul doute que les lecteurs des $A B P O$ suivent avec intérêt la progression du projet de recherche sur les sources hagiographiques de la Gaule antérieures à l'an Mil (SHG) programmé par l'Institut historique allemand de Paris. Annoncée dès la fin des années soixante-dix du siècle dernier et présentée en 1987 dans la revue Francia (Band 15, p. 701-731) par un article fondateur de ses trois promoteurs, François Dolbeau, Martin Heinzelmann et Joseph-Claude Poulin, cette entreprise de longue haleine a depuis donné régulièrement lieu à publications dont plusieurs ont fait l'objet de CR dans notre revue. En effet, à l'exception du dossier sur saint Géraud d'Aurillac dû à Anne-Marie Bultot-Verleysen, les quatre autres dossiers hagiographiques déjà publiés par Joseph-Claude Poulin concernent la "Province de Bretagne" (englobant les diocèses de Rennes - mais pas celui de Nantes - et de Dol ainsi que les six diocèses que " ce dernier siège essaya en vain, du $\mathrm{IX}^{\mathrm{e}}$ au XII ${ }^{\mathrm{e}}$ siècle, de conduire à se séparer de la province ecclésiastique de Tours "). Ce choix de l'hagiographie bretonne comme champ d'expérimentation n'a rien de fortuit. Les débats érudits et passionnés du début du $\mathrm{XX}^{\mathrm{e}}$ siècle avaient, d'une certaine manière, déblayé le terrain : le Memento des sources hagiographiques de l'histoire de Bretagne de l'abbé (et non "chanoine" - p. 235) François Duine qui s'en fait l'écho n'est toujours pas remplacé! Voici plusieurs dizaines d'années que des travaux pionniers (dont le plus retentissant est sans doute Les origines de la Bretagne de Léon Fleuriot) ont contribué "au réveil de la belle au bois dormant ", pour reprendre une jolie formule de Pierre Riché. En effet, comme vient de le remarquer par ailleurs François Dolbeau, l'un des animateurs des $S H G$, la Bretagne du haut Moyen Âge est peut-être la région " où les textes hagiographiques ont le plus d'importance par rapport au reste de la documentation médiévale. " Ce n'est donc pas un hasard non plus si le « dossier de saint Samson de Dol " par J.-C. Poulin a été proposé comme prototype des $S H G$. La controverse sur la date de rédaction de la première Vie de ce saint, tête de série ( $\mathrm{VII}^{\mathrm{e}}$ siècle ou $\mathrm{IX}^{\mathrm{e}}$ siècle ?) de l'hagiographie bretonne, est donc repartie allègrement, alors qu'elle s'était interrompue - 
faute de combattants - à la suite de la première guerre mondiale. Entretemps, Pierre Flobert a donné l'édition scientifique de La Vite ancienne de saint Samson de Dol qui faisait jusqu'à présent défaut (voir le CR dans les $A B P O$, t. 104, 1997/4, p. 100-103).

En effet, le regard porté sur les sources hagiographiques a été totalement renouvelé depuis un demi-siècle, si bien que la plupart des éditions disponibles s'avèrent très insatisfaisantes eu égard aux critères scientifiques actuels. C'est pourquoi, à défaut d'être en mesure de préparer les nouvelles éditions nécessaires, les collaborateurs des $S H G$ s'assignent du moins comme objectif d'établir " un bilan récapitulatif des connaissances sur l'attribution, la localisation et la datation des œuvres hagiographiques narratives en s'appuyant sur un réexamen méthodique de la tradition manuscrite " indispensable pour permettre l'exploitation de cette documentation à des fins historiques. À côté de monographies parues notamment dans Francia, la publication en 1992, sous la direction de Martin Heinzelmann de l'ouvrage collectif Manuscrits hagiographiques et travail des hagiographes a représenté une étape dans l'élaboration de la "méthode SHG " (voir le CR dans les $A B P O$, t. 100, 1993/3, p. 380-382). Toutefois, sur cinq études consacrées aux problèmes soulevés par la tradition manuscrite, seule celle de Joseph-Claude Poulin (" les dossiers des saints Melaine, Conwoion et Mervé ") représentait alors une contribution aux $S H G$ proprement dite. Le présent ouvrage - d'un volume presque deux fois plus important que le précédent - en inversant les proportions, atteste que l'entreprise a dorénavant atteint sa vitesse de croisière. Sur les six études présentées ici, quatre constituent les $S G H$ VI à IX qui concernent une cinquantaine de textes hagiographiques. S'y ajoutent deux contributions plus ponctuelles, qui font figure d'études préparatoires, en préalable à des dossiers à venir. Celle de Michèle Gaillard (" de l'Eigenkloster au monastère royal : l'abbaye Saint-Jean de Laon, du milieu du VII ${ }^{\mathrm{e}}$ siècle au milieu du VIII ${ }^{\mathrm{e}}$ siècle à travers les sources hagiographiques ") s'inscrit dans la ligne d'une réflexion sur le rôle de l'aristocratie dans les fondations monastiques durant le haut Moyen Âge. Elle démontre que les Vies rédigées à Laon reflètent, à deux moments bien distincts de l'ascension des Pippinides, l'intégration d'une fondation monastique féminine influencée par le mouvement colombanien dans le contexte politique et social contemporain. La contribution de Bruno Judic (" le culte de saint Grégoire le Grand et les origines de l'abbaye de Munster en Alsace ") s'attache minutieusement à détecter les indices de la diffusion précoce, en Alsace aux VII $\mathrm{e}_{-\mathrm{VIII}}{ }^{\mathrm{e}}$ siècles, du culte de saint Grégoire sous l'influence de la piété colombanienne relayée par les missionnaires anglo-saxons, avec l'appui des puissantes familles aristocratiques austrasiennes. Les Gesta des évêques de Toul (XII ${ }^{\mathrm{e}}$ siècle ?) apportent un témoignage, parmi d'autres, de l'empreinte de " la spiritualité de Colomban, mais aussi de Grégoire le Grand à travers Colomban " sur ces mêmes milieux. 
Le dossier constitué par Monique Goulet sur «les saints du diocèse Toul " conforte ce lieu-commun selon lequel la " quasi-totalité des saints du diocèse de Toul antérieurs au VIII ${ }^{\mathrm{e}}$ siècle sont réputés irlandais ". C'est, entre autres, le cas du premier évêque de ce siège, Mansuy (Mansuetus) et de son successeur Amon qui lui est toujours associé dans la littérature hagiographique. La Vita prolixior commandée dans le dernier quart du $\mathrm{X}^{\mathrm{e}}$ siècle par l'évêque de Toul Gérard à Adson de Montier-enDer s'inspire d'anciens Gesta episcopaux (perdus). Cette œuvre d'un hagiographe patenté est davantage un témoignage sur les méthodes de travail de celui-ci qu'elle n'apporte des informations sur les origines du diocèse de Toul et la personnalité de son fondateur. On sait que chez les auteurs du haut Moyen Âge, le terme Scoti désigne aussi bien les Bretons (insulaires, voire péninsulaires) que les Irlandais proprement dits. C'est pourquoi certains chercheurs sont tentés d'assimiler Mansuy de Toul au Mansuetus "évêque des Bretons " qui a assisté au concile de Tours de 461. Cette hypothèse n'est pas retenue par Monique Goulet qui n'exclut pas l'éventualité d'une confusion, tout en écartant l'hypothèse de Nancy Gauthier pour qui l'expression scripturae documento employée par l'hagiographe désigne les actes de ce concile. Par ailleurs, la présentation des sources hagiographiques concernant les saints de Remiremont ainsi que les autres saints vosgiens contribue à clarifier des questions longtemps embrouillées par les débats entre érudits locaux, laïcs ou ecclésiastiques, soucieux d'assurer la promotion de Remiremont.

Les deux dossiers consacrés par John Howe à l'hagiographie monastique de Haute-Normandie (SHG VII : "The hagiography of Jumièges (Province of Haute-Normandie) "; SHG VIII : " The hagiography of SaintWandrille (Province of Haute-Normandie) ") constituent la clef de voûte de l'ouvrage (p. 91-192). Ce sont des modèles du genre (60 \% des manuscrits mentionnés ont été directement consultés par l'A). Pour minutieuse qu'elle soit, l'étude des sources ne s'égare jamais dans des discussions oiseuses; l'examen critique de chaque pièce de ces deux dossiers conduit l'auteur à des conclusions d'ensemble qui réévaluent la production hagiographique en Neustrie durant la période mérovingienne. Ici encore, l'importance de l'influence du mouvement colombanien aux $\mathrm{VII}^{\mathrm{e}}$-VIII ${ }^{\mathrm{e}}$ siècles est mise en évidence. Quoique la Vita de saint Philibert, fondateur de Jumièges, soit dédiée à l'abbé Coschinus (qui semble avoir dirigé conjointement Jumièges et Saint-Riquier à la fin du $\mathrm{VII}^{\mathrm{e}}$ siècle), l'étude approfondie de celle-ci est reportée à un dossier ultérieur, à juste titre, dans la mesure où "the $S H G$ project specifies that saints will be situated in the region where the essentials of their dossier were redacted» et que c'est à partir de Tournus que s'est développé le culte de ce saint, à la suite de l'exode des reliques devant les invasions normandes. Signalons enfin, au passage, une précieuse mise au point sur saint Condède, dont la Vita fait figure de pièce rapportée dans la production hagiographique de Saint-Wandrille des premières décennies du $\mathrm{IX}^{\mathrm{e}}$ siècle. Léon Fleuriot ne déplorait-il que ce saint breton du $\mathrm{VII}^{\mathrm{e}}$ siècle 
soit "toujours oublié " dans l'étude des origines de la Bretagne, alors que son cas semble "indiquer la persistance d'éléments bretons [dans la région de la Basse Seine], peut-être jusqu'à la conquête normande "?

Avec les dossiers de saint Lunaire et de saint Paul Aurélien, JosephClaude Poulin poursuit sa présentation des sources hagiographiques bretonnes. Le signataire du présent C-R est en partie responsable de l'édition princeps de la Vita Ia mutilata $S$. Lenouerii. Ce n'est donc pas le lieu de débattre de la place tenue par une éventuelle Vita deperdita de saint Lunaire (abrégé d'une version primitive ou de la Vita IIa?). Nous y reviendrons ailleurs. Reconnaissons volontiers que l'étude codicologique mérite d'être approfondie; mais cela vaut aussi pour l'analyse linguistique des formes "très anciennes " de certains noms propres (cf. L. Fleuriot, $O B$, p. 280-281) qui, contrairement à ce qu'avance J.-C. Poulin, ne nous paraissent pas constituer une "base bien étroite pour asseoir une datation ancienne de la Vita» (p. 207, n. 21). S'il est évident que celle-ci se propose avant tout d'actualiser le message biblique et ne se soucie guère de la personnalité historique de son héros, il n'y a pas lieu pour autant, nous semble-t-il, de tergiverser sur le lieu de la rédaction primitive : certains récits de miracles destinés à garantir des donations ne fonctionnent que par rapport à la topographie de la paroisse qui a pris le nom de saint Lunaire! C'est pourquoi, ce texte nous paraît beaucoup moins "vide" que ne l'estime J.-C. Poulin. Cette divergence d'appréciation vaut, à plus forte raison, en ce qui concerne la portée historique de laVita de saint Paul Aurélien. C'est la seule source hagiographique bretonne dont l'auteur, le moine de Landévennec Wrmonoc précise la date de composition : 884. Malheureusement, ce texte n'est encore accessible que dans des éditions déplorables. Les recherches approfondies de F. Kerlouégan en préalable à une indispensable nouvelle édition (dont J.-C. Poulin annonce - p. 220 - qu'elle ne paraîtra finalement pas par les soins de ce chercheur, ce qui est regrettable!) ont néanmoins permis d'éclairer le vocabulaire, le style et les méthodes d'écriture d'un hagiographe qui s'est vu commander un " instrument d'entraînement scolaire " pour l'école épiscopale de Léon. Toutefois, cette destination de la Vita n'exclut pas, selon nous, que son auteur ait eu accès à de précieuses traditions (certes déformées selon les lois du genre hagiographique) relatives au rôle de la grande famille britto-romaine des Aureliani dans "l'histoire du VI ${ }^{\mathrm{e}}$ siècle".

Ainsi, l'expérience acquise par les promoteurs des $S H G$ les a conduit à étoffer leurs investigations en élargissant le cadre chronologique " antérieur à l'an Mil » défini au départ. Il s'avère que la réécriture constitue la "voie royale " des recherches en hagiographie, comme le constate Martin Heinzelmann dans sa préface. C'est ainsi que la Vita de saint Lunaire était considérée comme une composition tardive du XIII siècle par la plupart des spécialistes (à l'exception - notable - de Nora K. Chadwick et de Léon Fleuriot), jusqu'à ce que la mise au jour de fragments du $\mathrm{X}^{\mathrm{e}}$ siècle ne rende indispensable la prise en considération des réécritures 
postérieures. De même, pour ne retenir qu'un exemple dans la production hagiographique du diocèse de Toul, la Vita brevior de saint Mansuy (partie intégrante des Gesta pontificum Tullensium composés au XII siècle) est un remaniement des premiers Gesta épiscopaux (perdus) qui sont la source de la Vita prolixior composée par Adson de Montier-enDer. Les philologues ont sans doute beaucoup à tirer de l'analyse, à peine ébauchée, des procédés formels mis en œuvre pour amplifier ou pour abréger une œuvre. Mais, au Moyen Âge, la réécriture n'est pas un simple jeu littéraire : elle répond à des visées précises "glorifier Dieu, honorer les saints, enrichir le style des étudiants, transmettre les idées chères, préserver les biens de la communauté, adapter le texte au public " [Cf. P. Bouet, F. Kerlouégan, "la réécriture dans le latin du haut Moyen Âge", Lalies. Actes des sessions de linguistique et de littérature, 8 (Aussois, 26-31 août 1986) p. 161-163]. En fin de compte, c'est notre perception des mécanismes de pensée des lettrés médiévaux qui passe par l'attention portée aux recensions dérivées ou remaniées de ces Vitae. Il faut donc se féliciter de l'annonce par Martin Heinzelmann d'une prochaine série d'études portant sur la réécriture hagiographique.

Bernard Merdrignac

La ville médiévale en deçà et au-delà de ses murs. Mélanges offerts à JeanPierre Leguay. Textes réunis par Philippe LARDIN et Jean-Louis Roch, Presses Universitaires de Rouen, Rouen, 2000.

Il est tout à fait légitime que l'éminent spécialiste de l'histoire des villes de la fin du Moyen Âge et le non moins important spécialiste de l'histoire de la Bretagne qu'est Jean-Pierre Leguay se soit vu offrir par ses collègues des Mélanges centrés autour de ses deux passions. Les contributions consacrées à l'histoire des villes médiévales sont les plus nombreuses dans cet ouvrage. Elles évoquent tout d'abord les rapports de la cité avec son environnement à travers une grande variété d'exemples qui vont des Pyrénées (J. P. Barraqué) à l'Allemagne (P. Monnet) en passant par la Normandie (A. M. Flambard-Héricher, J. L. Roch). La société urbaine est étudiée dans toutes ses composantes, ses élites bien sûr (P. Cailleux, B. Leroy, A. Sadourny) mais aussi son peuple (J P Sosson, C. Vincent). La Bretagne se taille la part du lion dans cette évocation des villes de la fin du Moyen Âge et ce n'est que justice car elle fut le premier terrain de recherche de Jean-Pierre Leguay. À travers les contributions riches et originales de R. Cintré sur les réfugiés normands dans les villes bretonnes pendant la Guerre de Cent Ans, les avatars de la vie politique à Saint-Malo étudiés par A. Chédeville et $\mathrm{M}$. Jones et enfin, l'apparition des premiers galériens dans le port de Brest, découverts par J. Kerhervé. Ces travaux continuent et renouvellent les 